\title{
MS03-P22 | Crystal Structure Of Histone Chaperone Anti Silencing Function 1 (Asf1) From Plasmodium Falciparum And Its Biochemical Characterization
}

Kr. Srivastava, Dushyant (CSIR-Indian Institute of Chemical Biology, Kolkata, IND); Gunjan, Sarika (National Centre for Cell Science, Pune, IND); Seshadri, Vasudevan (National Centre for Cell Science, Pune, IND); Das, Chandrima (Saha Institute of Nuclear Physics, Kolkata, IND); Roy, Siddhartha (CSIR-Indian Institute of Chemical Biology, Kolkata, IND)

Chromatin structure and its dynamics are central to regulation of DNA template mediated cellular processes such as replication, transcription and repair. The chromatin structure continuously undergoes assembly and disassembly for facilitating access to nascent DNA sequence. This assembly and disassembly of chromatin is tightly governed and orchestrated by histone chaperone proteins. Anti silencing function 1 (Asf1) is a central histone chaperone which mediates deposition of histone $\mathrm{H} 3-\mathrm{H} 4$ into nucleosome structure in both replication dependent and independent manner. In this study we have done the structural characterization and molecular identification of Asf1 from Plasmodium falciparum (PfAsf1), which is the causative agent of severe form of malaria in humans. We have solved the high resolution crystal structure of histone chaperone domain of PfAsf1 protein. Two monomeric units of PfAsf1 molecule was observed in the asymmetric unit of the crystal structure of PfAsf1 indicating the protein might exists in a dimeric state which is different in comparison to its yeast and human homologues. This observation was further substantiated by in solution cross-linking experiments. Further, PfAsf1 interacted with histone $\mathrm{H} 3$ and $\mathrm{H} 4$ in our in-vitro protein interaction assay. Identification of critical residues mediating interaction of PfAsf1 and histone $\mathrm{H} 3-\mathrm{H} 4$ has been done and validated by site directed mutagenesis approach. Biophysical characterization of the PfAsf1-histone interaction was done by surface plasmon resonance (SPR) wherein high affinity interaction of PfAsf1 with $\mathrm{H} 3-\mathrm{H} 4$ was observed. The dimeric structure of PfAsf1 may be indicative of its divergent role in regulating the chromatin landscape in this pathogenic parasite.

Acta Cryst. (2019). A75, e73 